\title{
La Importancia del Dibujo en la Formación del Arquitecto: Equilibrio entre el diseño digital y el analógico
}

The Importance of Drawing in Architectural Formation: Balance between digital and analog design

\author{
Verónica Paola Rossado Espinoza \\ Universidad de Lima, Perú \\ vrossado@ulima.edu.pe
}

\begin{abstract}
There are many advantages that technology brings in support to the architect, among the skills that must be acquired by students of architecture, are, without a doubt, digital skills. However, excessive use of digital tools leads to lose other skills, such as drawing and hand design, with the consequent low quality and warmth of the artistic expression of the designer. It is important that academic training considers a balance between the application of technology and design, and that future professionals are as competent in both digital and analog design.
\end{abstract}

Keywords: Croquis; Arte; Competencias; Diseño arquitectónico; Expresión artística.

\section{Introducción}

La formación profesional en arquitectura, compromete la adquisición de una serie de competencias, habilidades y destrezas en determinadas áreas de especialización. Todo estudiante debe cultivar actitudes y valores en el transcurso de su vida académica, a fin de convertirse en un profesional competente. Con la aparición de la tecnología, surge la necesidad de dominar una nueva habilidad, conocida como "competencia digital", aplicada en muchos campos de la arquitectura, tanto en el trabajo proyectual como en la gestión de la construcción. En función a los avances y requerimientos del mundo actual, la estructura curricular debe incluir nuevos contenidos, basados en el nuevo perfil del egresado.

Con el uso de diversas herramientas digitales, el alumno puede ser capaz de desarrollar nuevas competencias relacionadas al diseño proyectual, como apoyo en el campo técnico y constructivo. Actualmente la adquisición de las competencias digitales va tomando mayor interés en escuelas de arquitectura e ingeniería. Sin embargo, es probable que el empleo excesivo de tecnología o "hiperconexión" en los estudiantes, interfieran con el logro de otras competencias esenciales para un arquitecto, como la habilidad de dibujar manualmente, fundamental como parte del proceso de diseño y expresión de ideas.

Este panorama trae consigo varias interrogantes: ¿Qué efecto produce el excesivo uso de tecnología en la práctica del diseño y el dibujo arquitectónico? ¿Cuáles serán las competencias digitales y analógicas que deben desarrollar los estudiantes de arquitectura según las exigencias del mundo actual?

A medida que la tecnología evoluciona, las carreras de arquitectura vienen utilizando nuevas aplicaciones en el diseño, representación y desarrollo de proyectos. Sin embargo, es posible que debido a un uso equivocado de los sistemas, los alumnos pierdan otras habilidades, como la sensibilidad artística y la interacción personal con la sociedad.

El presente artículo reflexiona sobre el efecto que ejerce la tecnología sobre la práctica del diseño arquitectónico y el dibujo a mano. Propone una estrategia que considera equilibrar el uso de herramientas digitales y analógicas dentro de la enseñanza - aprendizaje de la arquitectura. Mediante su aplicación, se pretende valorar la expresión artística del arquitecto, de manera que le permita expresar sus ideas en armonía con su entorno cultural, tecnológico y social.

\section{La Formación de Arquitectos}

Un egresado de arquitectura debe estar preparado para aplicar sus conocimientos en numerosas áreas de la especialidad, tales como: urbanismo, conservación, desarrollo y gestión de proyectos de construcción, a fin de generar un impacto positivo a los problemas de hábitat.

Durante su etapa de formación, el estudiante adquiere progresivamente el dominio de diversas competencias relacionadas al diseño arquitectónico, a través de su paso por las diversas asignaturas que forman parte de la estructura curricular de la carrera. Estas competencias le permitirán desarrollarse como un profesional capaz de adecuarse a cualquier situación o necesidad específica.

"Una persona competente es alguien que posee no sólo lo conocimientos y destrezas técnicas, sino también las capacidades prácticas o psicosociales requeridas por la situación". (Mastache 2007, pág. 79).

La competencia es la capacidad que se obtiene producto de la práctica, las habilidades, las actitudes, los valores y destrezas, en un ambiente motivado por aprender y obtener nuevo conocimiento. El competitivo mercado laboral necesita 
profesionales con visión de futuro y competentes, que puedan afrontar exitosamente los retos en su vida profesional.

El egresado debe estar en condiciones de responder eficientemente a las necesidades de un mundo exigente y tal vez adverso, adaptándose a cualquier ambiente laboral. Esta situación sugiere que el estudiante ejercite, durante su etapa de formación, diversas capacidades que le permitan resolver con resiliencia cualquier situación, impulsando su desarrollo y crecimiento personal.

\section{Las competencias en la arquitectura}

Un aprendizaje integral basado en competencias considera la adquisición de diversas habilidades y aptitudes que el alumno necesita para desempeñarse eficientemente en su vida profesional.

Entre las principales competencias necesarias para la carrera de arquitectura, se encuentran:

- Competencias básicas, son las relativas a las capacidades esenciales para todo estudiante, como las matemáticas, el cálculo, el lenguaje, la redacción, la comunicación, la ciencia, la cultura y el análisis.

- Competencias de formación, son las relativas con el desempeño de la profesión, como: creatividad, destreza manual, espacial, artística, visual, etc.

- Competencias técnicas y computacionales, están referidas al uso de instrumentos, herramientas o equipos informáticos, la capacidad de resolver problemas a nivel procedimental. Por lo general son derivadas de los avances tecnológicos.

- Competencias sociales, son las que permiten adecuarse y relacionarse con el entorno humano. Entre las más resaltantes están: la colaboración, la comunicación, la empatía, la cooperación, el respeto, la ética, la integridad y el trabajo en equipo.

- Competencias organizacionales, relacionadas a la capacidad de tomar decisiones, como: gestión, liderazgo, participación, organización e integración del equipo de trabajo.

En el campo de las competencias técnicas o computacionales, se encuentra la llamada competencia digital, que complementa la comprensión y adquisición de otras áreas de especialización. Es importante resaltar que, en la representación tridimensional y el dibujo de planos, inicialmente se utilizaba un software especializado, actualmente la computación aplicada a la arquitectura se ha extendido hacia innumerables aplicaciones.

"La competencia digital exige una buena comprensión y amplios conocimientos sobre la naturaleza, la función y las oportunidades de las tecnologías de la sociedad de la información (TSI) en situaciones cotidianas de la vida privada, social y profesional. Las capacidades necesarias incluyen: la capacidad de buscar, obtener y tratar información, así como de utilizarla de manera crítica y sistemática, evaluando su pertinencia y diferenciando entre información real y virtual, pero reconociendo al mismo tiempo los vínculos. Las personas deben ser capaces de utilizar herramientas para producir, presentar y comprender información compleja y tener la habilidad necesaria para acceder a servicios basados en Internet, buscarlos y utilizarlos, pero también deben saber cómo utilizar las TSI en apoyo del pensamiento crítico, la creatividad y la innovación."(Unión Europea, 2006, p.16)

"Las TIC se caracterizan por su interactividad, rapidez de acceso a una cantidad de información, instantaneidad e interconexión, cualidades que han de tenerse en cuenta para el desarrollo de la competencia digital." (Callejas, 2016, p. 685).

"La International Society for Technology in Education (ISTE) es una organización sin ánimo de lucro que tiene por misión promover la innovación en los procesos de enseñanzaaprendizaje con el uso de la tecnología. ISTE publicó los primeros Estándares Nacionales de Tecnología Educativa (NETS) para Estados Unidos en 1998. En 2006, ISTE ha propuesto una versión de los estándares NETS que se centra más en las habilidades y los conocimientos especializados y reduce la importancia de los aspectos instrumentales. (Vivancos, 2014, pág. 46).

Resumen de las dimensiones de los estándares NETS 2006:

- Creatividad e innovación: referida al pensamiento creativo, construyen conocimientos, desarrollan productos y procesos innovadores utilizando la tecnología.

- Comunicación y colaboración: relacionada con el uso de medios y entornos digitales para comunicarse y trabajar en forma colaborativa. Incluso a distancia, apoyan el aprendizaje individual y grupal.

- Investigación y fluidez informacional: aplicación de las herramientas digitales para obtener, evaluar y usar la información.

- Pensamiento crítico, resolución de problemas y toma de decisiones: para planificar y conducir la investigación usando herramientas y recursos digitales apropiados.

- Ciudadanía digital: relacionada con los asuntos humanos, culturales, sociales, practican conductas éticas y legales.

\section{- Conceptos teóricos y funcionamiento de la tecnología.}

Dentro de las dimensiones relativas a la adquisición de competencias, se incluyen los instrumentos digitales en la adquisición y construcción de nuevas habilidades, conocimientos y destrezas de formación, tanto sociales como organizacionales. 
Con el tiempo la tecnología ha tomado mayor importancia en el quehacer arquitectónico, extendiéndose en numerosas áreas de la especialidad. Es aplicada tanto en el campo del diseño paramétrico, como en la organización y en la gestión de proyectos, abriendo nuevas oportunidades para el trabajo en equipo. Es importante para el arquitecto, contar con una herramienta tecnológica como apoyo, un complemento para la expresión de sus ideas, sin interferir con su creatividad. En este entorno tecnológico, el diseñador debe tener la capacidad de manejar tanto las herramientas digitales como analógicas, de acuerdo a los requerimientos de cada situación.

En relación al entorno educativo en el aspecto digital, será necesario que el alumno adquiera diversas actitudes de responsabilidad y ética, ante la facilidad de acceso de información. La gran cantidad de proyectos arquitectónicos publicados en la web, pueden motivar el plagio y atentar contra la originalidad y la creatividad. Por la facilidad de acceso, en ocasiones los estudiantes obtienen una información equivocada y sin estudio previo. Es importante evaluar el origen de la información, reconocer y analizar la validez de cualquier publicación web antes de utilizarla.

"Es una problemática que, además de evidenciarse en las escuelas, se experimenta en nuestras ciudades, en las cuales constantemente encontramos copias de elementos que no son procesados ni pensados para nuestras condiciones, sino que simplemente están ahí porque se ven bien y parecen contemporáneos." (Castaño, Bernal y Cardona, 2005, p. 139)

\section{La Importancia de la Expresión Artística}

Un arquitecto es un artista en la creación de espacios, ningún software logrará representar las ideas con la habilidad artística y calidad del trazo del diseñador. La calidez del dibujo y la posibilidad de plasmar las ideas en cualquier momento, superficie o lugar, solo la puede realizar la mano humana.

Según Rafael Moneo: "A pesar de creer que la cultura digital llevará inevitablemente a nuevos modos de representación y de transmisión de la información acerca de cómo construir, la arquitectura se piensa siempre desde el dibujo... El arquitecto siempre hará uso del 'rasguño', de los croquis, de los apuntes espontáneos, que adelantan lo que un edificio puede ser". (Moneo, 2017)

Sin ánimos de desestimar las bondades de las herramientas digitales y su aplicación en la arquitectura y la ingeniería, es conveniente que la expresión del diseñador no esté supeditada a un determinado software o entorno digital. Como artista creativo, un arquitecto podrá utilizar cualquier material disponible para expresar sus ideas, ya sea un lápiz o un computador. "La manipulación de diversos materiales y herramientas para dibujar y construir modelos intensifican la percepción táctil y permiten completar el conocimiento a través de la visión". (Trachana, 2012, p. 291).

Con ayuda del software especializado, es posible analizar diversos factores de diseño, como en el caso de las estructuras, instalaciones eléctricas, sanitarias, lumínicas, acústicas y ambientales. A través de diversos programas es posible analizar el proyecto en conjunto, de igual forma, mediante el dibujo a mano, el diseñador puede comunicar gráficamente sus ideas, transmitiendo sensaciones a través del espacio, los materiales y acabados.

Grandes maestros de la arquitectura aplican tecnología en sus proyectos: "El estudio de Frank Gehry ha desarrollado incluso su propio software: el Digital Project, porque los que existían en el mercado no alcanzaban a dar respuesta a sus requerimientos de diseño. Sin estas herramientas hay edificios que no podrían haberse diseñado ni construido nunca. Basado en Catia, una herramienta desarrollada para el diseño aeronáutico, e implementando la tecnología BIM de diseño arquitectónico y cálculo paramétrico, el Digital Project ha permitido representar y materializar edificios como el Guggenheim de Bilbao, el Dongdaemun Design Plaza de Zaha Hadid o concluir la obras de la Sagrada Familia." (Borgesi, 2015).

"Por otro lado encontramos en los dibujos del arquitecto Oscar Niemeyer una síntesis formal en la construcción de bocetos. La línea limpia y segura con trazos amplios nos muestra una arquitectura casi orgánica y amable con el entorno. Niemeyer recurre a dibujos casi poéticos, sintéticos pero altamente descriptivos que nos dan una idea acabada del objetivo que persigue." (Amenedo, 2017, p. 117)

"La unión del ojo, la mano y la mente crea una imagen que no es solo un registro o una representación visual del objeto, sino que es el objeto mismo". "Dibujar es un proceso de observación y de expresión, de recibir y dar al mismo tiempo. Todo boceo y todo dibujo contienen una parte del creador y de su mundo mental, al tiempo que representa un objeto o un panorama del mundo real o de un universo imaginado." (Pallasmaa, 2012, p. 91 - 100).

Un dibujo a mano, o un croquis, plasma las sensaciones del diseñador en una superficie, es una forma fácil y rápida de representar ideas. "Un croquis debe alertar técnicamente y emocionar plásticamente al observador. Debe transmitir sensaciones imposibles de informatizar, siendo de ejecución rápida, permite prever las posibles fallas de un proceso..." (Quintana, 2009, p. 25).

La solución está en el equilibrio, tanto el uso de un software adecuado, como el uso del lápiz en el diseño, son esenciales para que el arquitecto comunique claramente sus ideas.

\section{Las herramientas digitales en el dibujo}

Existen numerosos programas aplicados a la arquitectura, ingeniería y construcción, que son necesarios para la realización de un proyecto arquitectónico integral, pero no existe un software específico que pueda adaptarse a todas las necesidades o panoramas de diseño. Dependerá del diseñador la elección de la más adecuada, algunas veces será necesario analizar cuestiones técnicas, estándares del país, recursos y compatibilidades, para optimizar el diseño arquitectónico y la gestión del proceso constructivo.

Del mismo modo, es importante mantener un uso responsable del internet a nivel académico, algunos estudiantes, ya sea por 
inexperiencia o facilismo, tienden a buscar respuestas a cualquier problema en páginas web, tomando ejemplos inadecuados como modelo para sus trabajos. Esa situación obliga a los docentes a reforzar en sus alumnos normas éticas y de conducta, que le permitan impulsar su capacidad creativa y de investigación con ayuda de los medios de comunicación.

"Desde la Universidad tenemos que favorecer las buenas prácticas investigadoras que favorezcan el correcto uso de la información, el respeto de la propiedad intelectual y a la legalidad vigente. El problema surge porque la tecnología facilita estas prácticas y falla la metodología de la enseñanza - aprendizaje, que lleva al estudiante a tener que responder un sinfín de trabajos académicos sin que se le haya formado de forma previa en las habilidades y destrezas necesarias". (Alfaro y Juan, 2014, p. 5)

Lamentablemente el mundo digital trae consigo avances y tropiezos, queda en manos de los educadores controlar el manejo de los recursos digitales, ejercitando virtudes de responsabilidad y profesionalismo. Los estudiantes deben cultivar buenos valores y hábitos de investigación con recursos tecnológicos, del mismo modo que sucede con el empleo de libros o revistas. Es posible construir soluciones innovadoras y creativas, a partir del análisis y el estudio de obras y el estilo arquitectónico de los grandes maestros, sin cometer plagio o atentar contra la propiedad intelectual o el patrimonio edificado.

La creatividad, el compromiso con la sociedad, el respeto y la investigación, serán claves en la formación de un profesional exitoso. Es importante que el docente aplique una adecuada estrategia de enseñanza - aprendizaje, empleando con responsabilidad todos los recursos disponibles, tanto tecnológicos como analógicos, motivando al estudiante en la adquisición de competencias técnicas, sociales y de especialidad. Será esencial preservar la sensibilidad y la habilidad artística del estudiante, evitando el excesivo uso de herramientas digitales en el proceso de aprendizaje y ejercicio de la arquitectura.

\section{El área académica de comunicaciones}

Diversos contenidos de las asignaturas formativas de la Facultad de Ingeniería y Arquitectura, exigen el dominio de herramientas digitales, pero su aprendizaje se realiza de manera gradual. En las asignaturas de talleres de diseño, la facultad pone especial interés en las técnicas de expresión manual y artística, donde los alumnos utilizan distintas formas de expresión para desarrollar maquetas y paneles. En el área de comunicaciones, en las diversas asignaturas como dibujo y comunicación, el alumno adquiere el dominio de distintas técnicas manuales y digitales. Utilizan los recursos gráficos para lograr comunicar con claridad las propuestas arquitectónicas, utilizando las diferentes formas de expresión, composición y presentación digital.

Con la reciente introducción del concepto BIM (modelado de la información) el diseñador maneja una metodología de trabajo colaborativo, la cual es empleada como una plataforma que facilita la gestión, el análisis estructural, las instalaciones y la organización en general del proyecto de construcción. Es una metodología que viene siendo introducida por muchos profesionales con excelentes resultados, sin que necesariamente interfiera en la labor proyectual del arquitecto. Es una plataforma que permite la coordinación entre los diferentes agentes involucrados, optimizando el proyecto integral.

Es importante reconocer que el futuro arquitecto debe ejercitar su habilidad en resolver problemas de diseño considerando diversos factores, como ambientales, de entorno, de construcción y entender las necesidades de los usuarios, siendo importante aplicar una herramienta de soporte tecnológico que permitan analizar dichas condicionantes.

Las competencias que deben adquirir los estudiantes de arquitectura, establecen tanto el dominio de herramientas digitales como analógicas, que le permitan manejar con destreza cualquier entorno digital, sin perder sus habilidades de dibujo manual. Un alumno debe ser capaz de expresar sus ideas tanto de manera análoga como digital, eligiendo la estrategia que se adecúe mejor a su forma de trabajo, en función a las condicionantes y necesidades de cada situación.

"La mano capta la cualidad física y la materialidad del pensamiento y la convierte en una imagen concreta. En el arduo proceso de proyecto, la mano a veces toma las riendas en el sondeo de una visión, con un vago presentimiento de que finalmente se convertirá en boceto, en una materialización de una idea. En manos del arquitecto el lápiz constituye un puente entre la mente que imagina y la imagen que aparece en la hoja de papel..." (Pallasmaa, 2012, op cit. p. 12-14).

"El dibujo a línea, más abstraído que sólo el dibujo real, no sólo tiene la capacidad de facilitar la transmisión de la IDEA central, sino también de aclarar de una sola vez todo lo que nos proponemos transmitir." (Campo, 2009, p. 74).

\section{Propuesta de Equilibrio entre el Diseño Digital y Analógico}

En la adquisición de competencias de formación del estudiante de arquitectura, es imprescindible olvidar por un momento el CAD, las TIC, el BIM y el diseño paramétrico, dado que la habilidad proyectual no está limitada a un software. Es necesario que el alumno pueda aprender a usar las herramientas digitales aplicadas al diseño de manera responsable y oportuna, en combinación con técnicas manuales, a fin de que éstas no entorpezcan con el desarrollo de la creatividad.

La propuesta promueve la adquisición de competencias desde un enfoque conceptual (saber), procedimental (saber hacer) $y$ actitudinal (querer hacer), afrontando con resiliencia las presiones de un mundo tecnológico y encontrando soluciones creativas a cualquier problema. Los alumnos deben aprender a expresar libremente sus ideas, en función a los problemas planteados, las necesidades de la sociedad y su entorno.

Se recomienda la práctica académica del diseño arquitectónico desde tres aspectos: la creatividad (durante el proceso de diseño), la expresión gráfica (para la representación de ideas) y la interacción sociocultural (aproximación con las personas y el ambiente). 
A fin de cumplir con estos objetivos, se propone que la formación esté fundamentada en los siguientes perfiles:

Desde el aspecto conceptual:

- Ejercitar el pensamiento crítico.

- Aplicar conocimientos previos.

- Analizar variables y condicionantes de diseño.

- Aplicar métodos de indagación e investigación.

- Desarrollar el pensamiento reflexivo.

- Analizar diversas alternativas de solución.

Desde el aspecto actitudinal:

- Trabajar en equipo.

- Practicar técnicas de liderazgo y colaboración.

- Usar responsablemente la información.

- Asumir riesgos.

- Aceptar críticas.

- Exponer y defender las ideas.

- Reforzar la autonomía y el autoaprendizaje.

- Mantener interés por el tema.

- Mantener asistencia constante y puntualidad.

- Demostrar integridad, respeto y ética.

Desde el aspecto procedimental:

- Ejercitar el dibujo manual para la comunicación.

- Organizar croquis y esquemas.

- Experimentar distintas texturas y materiales de acabados.

- Buscar y analizar diversas soluciones.

- Evaluar alternativas.

- Desarrollar y replantear en base a las críticas.

- Expresar ideas mediante apuntes y bocetos.

- Ejercitar adecuadamente las instrucciones o comandos dentro de un sistema computarizado.

\section{La formación integral del estudiante}

El plan de estudios propone un equilibrio entre las ciencias, tecnología, artes, humanidades y gestión empresarial. El contenido temático de las asignaturas que forman parte de la estructura curricular, considera la adquisición de habilidades digitales que complementan las competencias de formación, así como de los componentes artísticos y culturales.

El ejercicio de un proyecto académico, debe reforzar en los alumnos las siguientes competencias:

a) De relación con el desarrollo sostenible:

- Compromiso con la sociedad.

- Relación con su entorno.

- Respeto por el medio ambiente.

- Responsabilidad social y personal.

- Compromiso con el patrimonio edificado.

- Respeto a los monumentos arqueológicos.

b) De colaboración:

- Conciencia de grupo.

- Apoyo mutuo.

- Planificación de actividades.

- Trabajo multidisciplinario.

- Coordinación del equipo de trabajo.
- Igualdad de responsabilidades.

- Comunicación eficaz.

- Organización.

- Liderazgo.

b) De creatividad e innovación

- Pensamiento crítico y analítico.

- Investigación.

- Autoaprendizaje.

- Visión de futuro.

- Innovación y creatividad.

- Trabajo autónomo.

- Artísticas.

- Culturales.

- Aplicación de conocimientos previos.

c) De investigación científica y tecnológica

- Toma de decisiones.

- Perfeccionamiento.

- Dinamismo.

- Perseverancia en la búsqueda de objetivos.

- Organización de actividades.

- Técnicas de representación.

d) De ética profesional:

- Integridad.

- Respeto.

- Emisión de juicio crítico.

- Responsabilidad.

- Respeto de los derechos de autor.

- Adaptabilidad a los cambios.

- Ética profesional.

\section{Recomendaciones}

La estructura curricular y la metodología de enseñanza aprendizaje deberá permitir al alumno aprender en base a competencias, de manera que pueda adquirir conocimientos desde un enfoque conceptual (saber), procedimental (saber hacer) y actitudinal (querer hacer), afrontando con resiliencia las adversidades y resolviendo exitosamente cualquier problema, ya sea durante su vida académica o profesional.

El contenido de cada asignatura, deberá comprender los aspectos conceptuales, actitudinales y procedimentales necesarios para el ejercicio del diseño y la expresión arquitectónica, mediante el desarrollo de habilidades de comunicación manuales y digitales. El docente deberá mantener el equilibrio de dichas competencias, de manera que el alumno tenga la oportunidad de expresarse analizando diversas condicionantes arquitectónicas, sin olvidar las necesidades del usuario, de manera que mantenga siempre un contacto con su entorno, la localidad y la sociedad.

El docente deberá promover la adquisición de los diferentes valores a lo largo de la formación académica, donde el estudiante refuerce la práctica responsable de diseño, ejercite su expresión a mano alzada y domine el software de su especialidad. Debe ser capaz de aplicar y seleccionar adecuadamente las herramientas digitales para analizar las 
condicionantes de diseño, así como representar sus ideas sin limitaciones.

Se sugiere mantener el énfasis en el dibujo manual, el croquis, el apunte y el boceto, aplicadas en la comunicación creativa de ideas, realizando ejercicios en un espacio físico exterior, que permita al alumno un mayor contacto con su entorno y las personas. Del mismo modo, deberá utilizar responsablemente las herramientas digitales en la investigación, organización y gestión del proyecto de construcción. Durante la práctica del diseño, es importante ejercitar el trabajo colaborativo, asegurando el dominio integral de todas las competencias de la carrera de arquitectura.

\section{Conclusiones}

Las competencias se construyen y se forman a través de la práctica, mediante situaciones o problemas que obliguen al alumno a tomar decisiones de nivel profesional, evaluando las posibilidades que le brindan las herramientas tecnológicas y análogas que tiene a su disposición.

En un mundo informatizado, el egresado de arquitectura, deberá asumir una actitud responsable en el uso de los medios digitales y la expresión artística manual. Existirán ocasiones que demanden el uso de la tecnología para la realización del proyecto, así como la expresión manual para comunicar rápidamente sus ideas. Está en manos del diseñador no poner límites a su creatividad y la elección de la herramienta más adecuada para cada situación.

El futuro arquitecto debe ser un exitoso profesional del siglo $\mathrm{XXI}$, dominar el diseño colaborativo en entornos digitales mediante la metodología BIM, e innovar en el diseño a través del lápiz. Un arquitecto no debe perder su habilidad artística, que le permitirá expresarse con creatividad mediante cualquier instrumento y en cualquier lugar.

\section{Agradecimientos}

Es propicio agradecer a los colegas que apoyaron la presente investigación, docentes integrantes de un excelente equipo de trabajo en la Facultad de Ingeniería y Arquitectura, de la Universidad de Lima.

\section{Referencias}

Alfaro, P. Juan, T. (2014). El Plagio académico: formar competencias y buenas prácticas universitarias. Revista de Unidades de Información. Número 6. RUIDERA. Universidad de Castilla - La Mancha. España.

Amenedo, G. (2014). El dibujo a mano alzada como herramienta en el proceso de diseño. Reflexión académica en diseño \& comunicación. I Congreso de creatividad, diseño y comunicación para profesores y autoridades de nivel medio. Universidad de Palermo. España. Recuperado de:

http://fido.palermo.edu/servicios_dyc/publicacionesdc/archivos/4 83_libro.pdf

Baeza, A (2009). Pensar con las manos. Editorial Nobuko. Buenos Aires. Argentina.

Borguesi, C. (2015). Arquitectura y representación. Página web: Arquitectos, diseñadores y creativos. Recuperado de: http://proyectodigital.com/arquitectura-y-representacion

Castaño, J., Bernal, M., Cardona, D. (2005). La enseñanza de la arquitectura: una mirada crítica. Revista Latinoamericana de Estudios Educativos. Bogotá. Colombia

Callejas, A., Salido, J., Jerez, O. (2016). Competencia digital y tratamiento de la información: aprender en el siglo XXI. Cuenca. Ediciones de la Universidad de Castilla - La Mancha. España.

Carbajal, A. (2006).Creatividad y construcción arquitectónica de vanguardia: estudio sobre proceso de invención y modelo didáctico de aplicación para el desarrollo creativo en la enseñanza aprendizaje en la introducción en el diseño arquitectónico. Universidad Complutense de Madrid. España.

Correal, G. (2011). Sobre modelos pedagógicos y el aprendizaje del proyecto arquitectónico: proyecto arquitectónico y urbano. Revista de Arquitectura, Vol. 13. Red Universidad Católica de Colombia.

Esquinas, F. (2011). Dibujo: artes plásticas y visuales: complementos de formación disciplinar. Ministerio de Educación de España Editorial GRAÓ, de IRIF, S.L. Madrid, España.

Flores, A., Grané, J. (2012). La Resiliencia en entornos socioeducativos. Narcea. Madrid, España.

Gutiérrez-Martín, A., Palacios-Picos, A., Torrego-Egido, L. (2010). Tribus digitales en las aulas universitarias. Comunicar, 34, XVII, 2010. Madrid.

Hernández, L., Miret Establés, E., Sancho, M. (2013). Representación del espacio arquitectónico. Prensas de la Universidad de Zaragoza. España.

Labarta, C., Bergera, I. (2011). Metodología docente del proyecto arquitectónico. Zaragoza. Prensas de la Universidad de Zaragoza. España.

Labarta, C. (2012). Proyecto arquitectónico y docencia: una cuestión de orden. Zaragoza. Prensas de la Universidad de Zaragoza. España.

Litwin, B., Sorondo, R., Uriburu, J. (2009). Pasos hacia una metodología de diseño. Buenos Aires. Editorial Nobuko. Argentina.

Mastache, A. (2007). Formar Personas Competentes. Desarrollo de competencias tecnológicas y psicosociales. Ediciones Novedades Educativas. Buenos Aires, Argentina.

Marciales, G., Cabra, F., Castañeda-Peña, H. (2013). Nativos digitales: transiciones del formato impreso al digital. Editorial Pontificia Universidad Javeriana. Colombia.

Méndez, A. (2006). Aprender modelando... Crear aprendiendo. Red Ensaios e Ciência. São Paulo, Brasil.

Mesa, Y. (2015). Modelo didáctico de la formación de las habilidades profesionales en la asignatura dibujo de construcción. Editorial Universitaria, 2015. Habana, Cuba.

Moneo, R. (2017). ABC Cultura. Artículo: La arquitectura se piensa siempre desde el dibujo. Recuperado de: http://www.abc.es/cultura/cultural/abci-rafael-moneoarquitectura-piensa-siempre-desde-dibujo201704020050 noticia.htm

Montero, L., García-Salazar, J., Rincón-Méndez, L. (2009). Una experiencia de aprendizaje incorporando ambientes digitales: competencias básicas para la vida ciudadana. Universidad de la Sabana. Bogotá. Colombia.

Pallasmaa, J. (2014). La mano que piensa: Sabiduría existencial y corporal en la arquitectura. Barcelona: Gustavo Gili.

Quintana, J. (2009). El croquis arquitectónico. Editorial Nobuko. Buenos Aires, Argentina. 
Rodríguez, D. (2014). Diseño Arquitectónico. Procesos del pensamiento gráfico. Editorial Universitaria. Universidad de Guadalajara. México.

Ruiz, N. (2013). En los Límites de la Arquitectura. Espacio, sistema y disciplina. Tesis de doctorado. Universidad de Cataluña. Barcelona.

Sainz, J. (2005). El dibujo de arquitectura. Teoría e historia de un lenguaje gráfico. Editorial Reverté. Barcelona. España.

Sierra, J. (1997). Manual de dibujo de la arquitectura, etc. Contra la Representación. Instituto Universitario de Ciencias de la Construcción. Sevilla. España.
Tachana, A. (2012). Manual o Digital. Fundamentos antropológicos del dibujar y construir modelos arquitectónicos. EGA Expresión Gráfica Arquitectónica. N. 19. Universidad Politécnica de Valencia. España. Recuperado de: https://polipapers.upv.es/index.php/EGA/article/view/1381

Unión Europea. (2006). Recomendación del Parlamento Europeo y del Consejo. Sobre las competencias clave para el aprendizaje permanente. Diario Oficial de la Unión Europea. España.

Vivancos, J. (2014). Tratamiento de la información y competencia digital. Madrid. Difusora Larousse - Alianza Editorial. España 